\title{
METROLOGY AND SIMULATION OF CHEMICAL TRANSPORT IN MICROCHANNELS
}

\author{
P. M. St. John*, T. Woudenberg, and C. Connell \\ PE Applied Biosystems \\ 850 Lincoln Centre Drive \\ Foster City, CA 94404
}

\author{
M. Deshpande and J. R. Gilbert \\ Microcosm Technologies, Inc. \\ $2151^{\text {st }} \mathrm{St}$ \\ Cambridge, MA 02142
}

\author{
M. Garguilo and P. Paul \\ Sandia National Laboratory \\ Livermore, CA
}

\author{
J. Molho, A. E. Herr, T. W. Kenny, M. G. \\ Mungal \\ 551 Terman \\ Stanford University \\ Stanford, CA 94305
}

fragments out to several kilobases but device area must be minimized. Flow predictions are also useful for determining the geometries needed for chemical reactions in microchannels.

\section{EXPERIMENTAL}

Silicon microchannel structures were fabricated as "negative" masters using standard photolithography and an STS deep reactive ion etch (DRIE) was used to create raised rectangular structures with dimensions of $50 \mu \mathrm{m}$ in width, $5 \mathrm{~cm}$ in length and $40-80 \mu \mathrm{m}$ in height. An RTV silicone elastomer (Dow Corning) was used to form positive replicas of the silicon structures (Fig. 1) [5]. Scanning electron micrographs (SEMs) show the etched silicon sidewalls and the corresponding elastomer replica (Au sputtered) (Fig. 2). The striae located near the top of the silicon structure was a result of over-etching. The etched silicon region was smooth to an RMS roughness of $7.26 \mathrm{~nm}$, determined by atomic force microscopy. The elastomer SEM shows the reproduction of the features present in

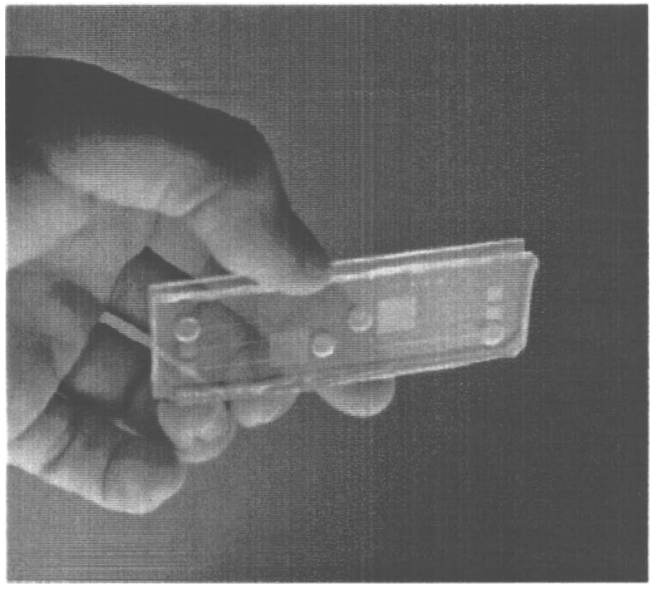

Figure 1. Photograph of the elastomer device with three test microchannels. The circles are the injection wells and the squares are used to study pressure driven flow in a sudden expansion. 


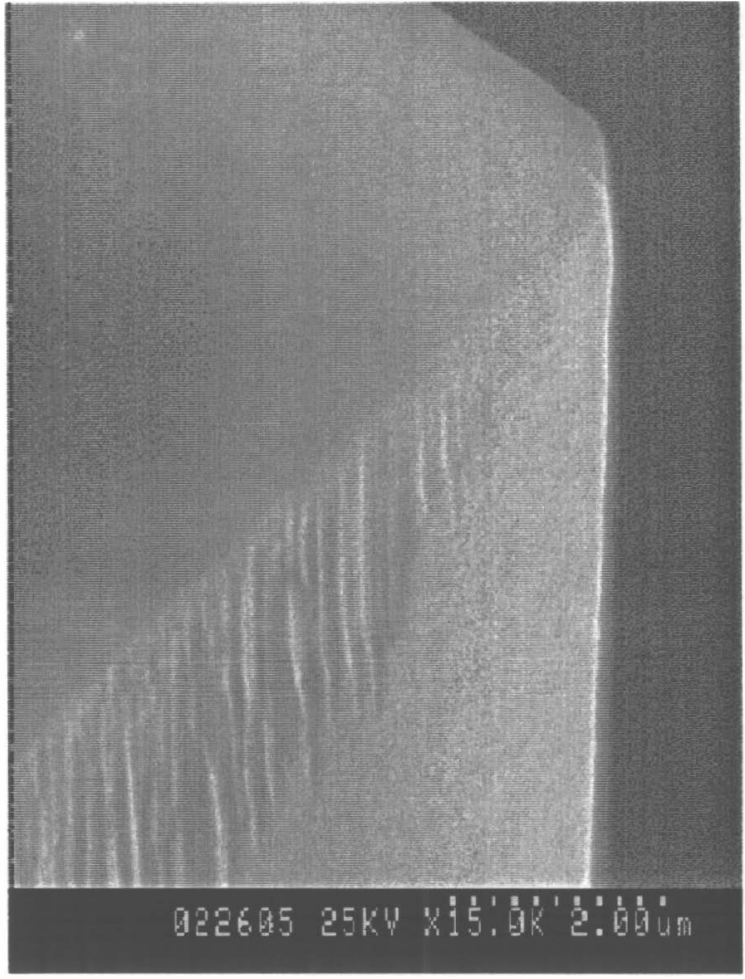

Figure 2a. SEM of the silicon mold

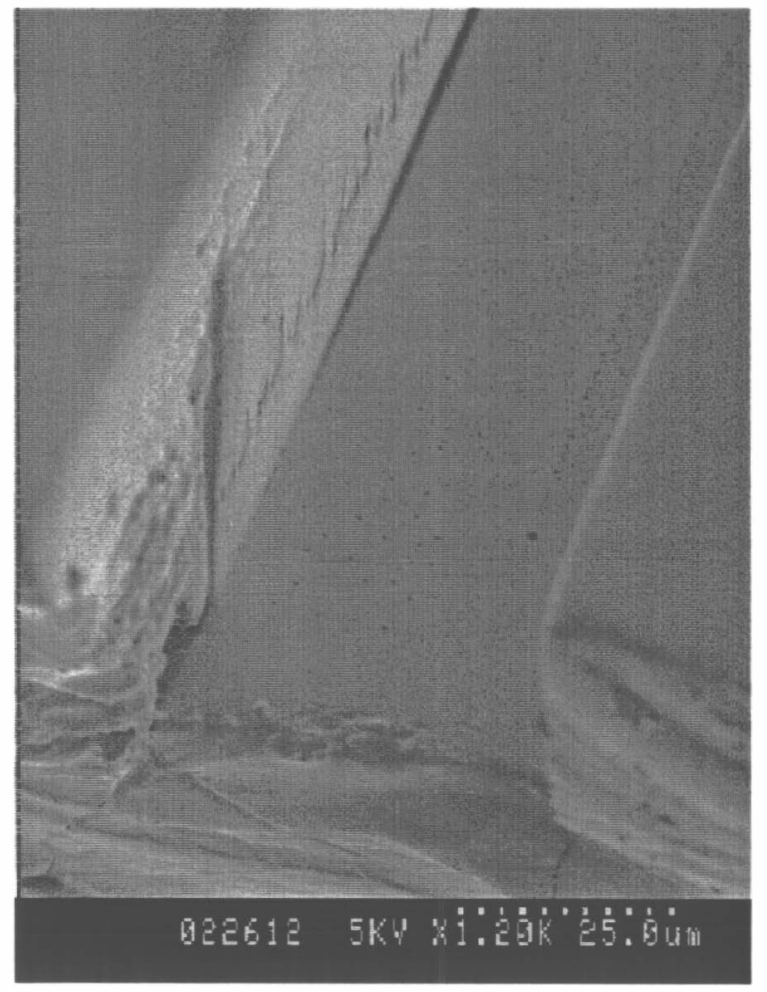

Figure 2b: SEM of the elastomer channel

the silicon. Note the striations which run the length of the channel on the lower portion of the channel wall. This corresponds to the rough overetched region of the silicon mold. The region of the elastomer which replicated the etched silicon surface had an RMS roughness of $\sim 7 \mathrm{~nm}$. The rough edge at the opening of the elastomer channel came from slicing the elastomer structure with a razor blade. The channel was not cleaned prior to metal sputtering and particulates can be seen on the channel floor. The elastomer channels were sealed with either glass microscope slides or elastomer coated glass microscope slides and wells were created in the elastomer using a mechanical hole punch (Figure 1).

Serpentine microchannels in borofloat glass (not shown) were fabricated by Alberta Microelectronics Centre (Alberta, Canada) using standard photolithography and an isotropic wet etch to yield a smooth hemispherical cross-section. A commercially available additive made from a polyacrylamide short chain polymer (ABI DNA Fragment Analysis Reagent 7\% $\mathrm{w} / \mathrm{w})$ was used $(0.1 \% \mathrm{v} / \mathrm{v}$ aqueous) to suppress electroosmotic flow (EOF) [6] in both glass and elastomer structures. Although EOF suppression was verified by observing ion travel under an applied field, the extent of suppression was not quantitated. In this paper, electrophoretic flow refers to negatively charged dye migration towards the positive electrode and electroosmotic flow refers to dye carried toward the negative electrode, despite charge.

The caged fluorophore (fluorescein) was purchased from Molecular Probes and used in micromolar quantities dissolved in either distilled water or Tris-EDTA buffer, $\mathrm{pH}=7.4$. The dye is negatively charged, both caged and uncaged (fluorescein, -2). When caged, the dye solution is non-fluorescent. Upon photoactivation of a UV light illuminated volume, the protecting group is cleaved and the dye fluoresces. Approximately $0.3 \mathrm{~mW}$ average power of $355 \mathrm{~nm}$ light from a pulsed $\mathrm{Nd}$ :YAG laser (Uniphase) was used for $\sim 0.1 \mathrm{~s}$ to uncage the fluorophore in a $20 \mu \mathrm{m}$ spot. In the electrokinetic experiments, the laser beam was focused using a UV microscope objective which defined a sharp start zone. A continuous wave Microblue diode pumped laser at $473 \mathrm{~nm}$ (Uniphase) was used for fluorescence excitation (Figure 3). The Microblue was positioned close to Brewster's angle for glass to minimize laser scatter into the microscope. Pressure driven flow was imaged at Sandia National Laboratories where the experimental setup is similar to that described above with the exceptions that the uncaged region is rectangular in shape and that the uncaging time is much less than one millisecond. Fluorescence images of the molecules diffusing and moving in the local flow were collected using a microscope objective (total magnification $=100 \mathrm{X}$ ) and a video rate, interlaced camera. Images were analyzed and flow parameters were extracted for simulations. The evolution of the fluorescent profiles under electrokinetic and pressure-driven flow are shown here by comparing a sequence of timed images to corresponding times from the simulations.

The numerical simulations were performed using the NetFlow module in Memcad [7]. It employs a three-dimensional finite element based tool to solve the Navier-Stokes equations. The electrokinetic effects can be broadly divided into two categories: electrophoresis, involving the transport of a charged species through a carrier due to a differential in the mobility, and electroosmosis, involving the pumping of the carrier fluid due to wall based effects. Electrokinetic effects were incorporated by coupling the Navier-Stokes equations with the Poisson equation for the electric field and with the Poisson-Boltzmann equation for the zeta potential in the electroosmotic cases [8].

The equations are solved here under the assumption of a dilute solution i.e. the carried species does not affect the material properties of the carrier. This allows the equations to be solved in their incompressible form. The finite element meshes were chosen generally to allow for adequate resolution of the 
boundary layer and accurate representation of all the experimentally observed fluid physics.

In the design of practical micro-devices, however, accurate coupling of the electrokinetic effects requires the knowledge of several material and physical parameters, such as the diffusivity and the mobility of the solute in the solvent and extent of surface charge on the inner walls of the microchannels. Additionally wall-based effects such as chemical binding sites might exist that affect the flow patterns. Our approach is to extract the required material parameters through quantitative comparison with experimental measurements in simple geometries and use the extracted parameters for simulations and comparisons in more complex geometries.

\section{Schematic of the uncaging apparatus}

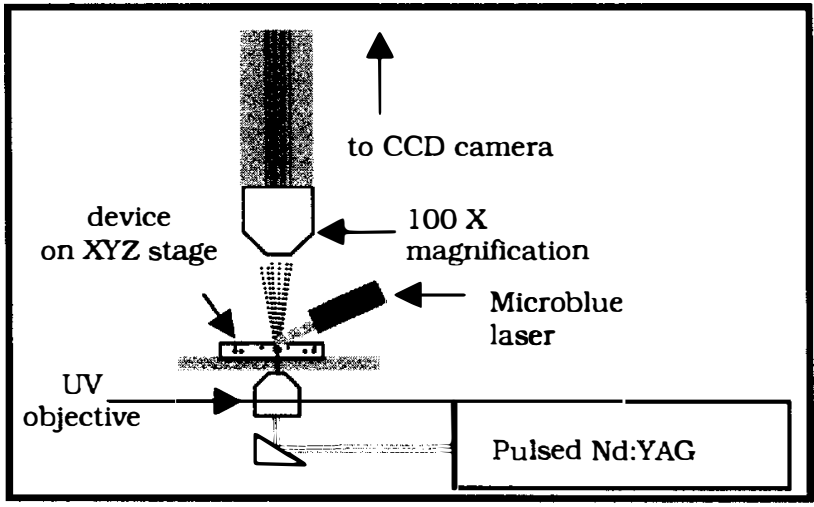

UV pulse initiates dye

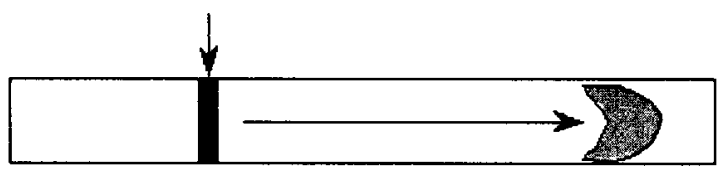

time evolved

Figure 3. Top: Schematic of the experiment. The Nd:YAG laser is used to uncage the dye and a Microblue $473 \mathrm{~nm}$ laser is used to excite the fluorescence. Bottom: Illustration of the uncaging process. The starting fluorescent fluid volume is defined by the uncaging laser (either as a spot or as a ribbon).

\section{RESULTS AND DISCUSSION}

Figure 4 shows a timed series of pressure-driven flow in microchannels which expand into a large planar reservoir. This figure demonstrates that the simulation qualitatively reproduces the dye dispersion from laminar flow seen during the experiment. The channel width was $100 \mu \mathrm{m}$ by $40 \mu \mathrm{m}$ deep. The flow rate was approximately $15 \mu \mathrm{L} / \mathrm{hr}$.

The Reynolds number, $\mathrm{Re}$, in this experiment is typical for bioanalytical systems $(\operatorname{Re}=0.05)$ although, it appears low when compared to typical numbers in micropumps $(\operatorname{Re} \sim 100)$. The images therefore differ from what on might expect to see at higher Reynolds number. The governing equations become linear and harmonic which results in the flow pattern appearing as if it evolved from a point source in Fig. 4. The experiments and simulations in the figure show good agreement which is to be expected for low Reynolds number flows. Similar experiments and stimulations were conducted on various other geometrical microchannel shapes and serve to verify the capabilities of our module in the simulation of pressure-driven flows.

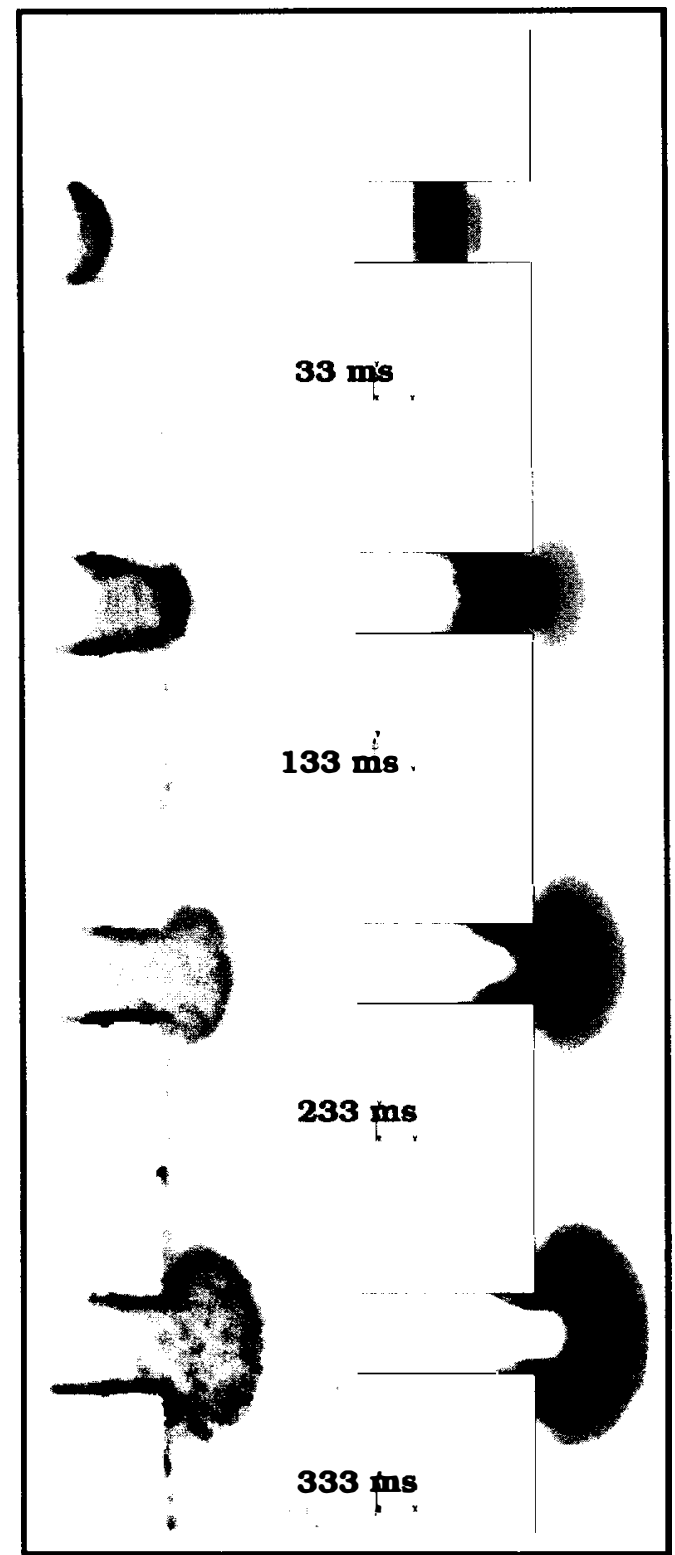

Figure 4. Experimental and simulated pressure-driven flow entering a sudden expansion in an elastomer structure.

Electrokinetic measurements using a range of applied fields and in a number of geometries were compared to simulations of electroosmotic and electrophoretic flow in both elastomer and glass microchannels. Figure 5 shows the simple case of a time-lapse experimental electrophoretic flow at a field strength $(E)=85.8 \mathrm{~V} / \mathrm{cm}$ in a $50 \mu \mathrm{m}$ straight elastomer channel with a $0.1 \%$ flowable and water soluble polymer added to the dye solution to suppress EOF [6]. For the case of electrophoretic flow where EOF is assumed negligible, the simulated and experimental band shape are similar. The simulated image (right) shows some of the features of the NetFlow software. This image is a 2-D histogram of the integrated experimental fluorescent profile for $t=0.99 \mathrm{~s}$. The 2D histograms provide crucial information regarding the surface forces acting on the flow. The simulation shows an intense 


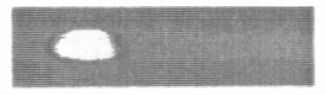

$\mathrm{t}=0.33$

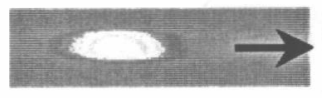

$\mathrm{t}=0.99$
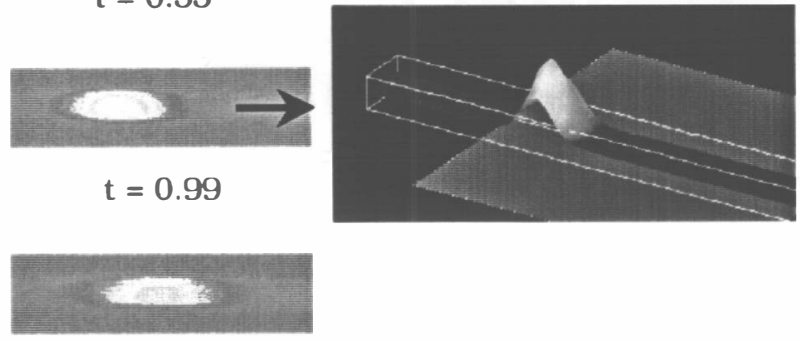

$t=1.65$

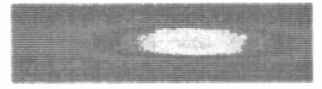

$\mathrm{t}=\mathbf{2 . 3 1}$

Figure 5. Left: Experimental electrophoretic flow in an elastomer channel, $E=85.8 \mathrm{~V} / \mathrm{cm}$. Right: Column integration tool from NetFlow used to form 2-D image/histogram corresponding to the fluorescence.

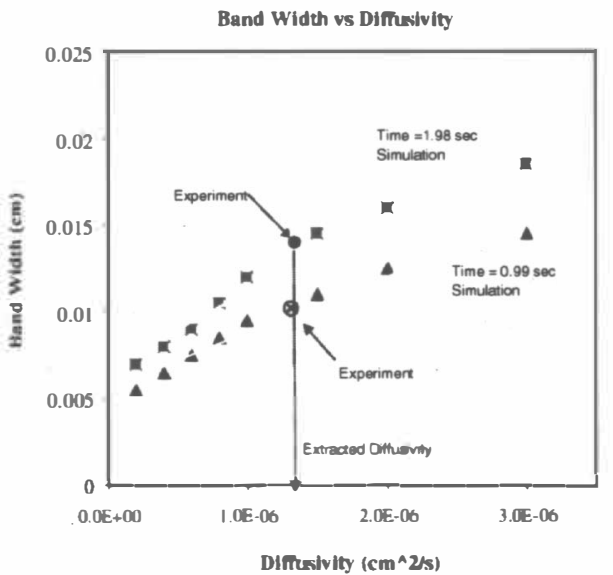

Figure 6. Graph of the simulated band width at two distinct times (squares and triangles) vs. a range of diffusivities. Experimental data (circles) were added to the plot to extract the diffusivity

center region (high mass fraction) and regions of very low intensity along the channel walls, implying that the fluorescent plug may be repelled in this region. The band broadening occurs rapidly because the low mass dye has a high diffusion constant, $\sim 1.3 \times 10^{-6} \mathrm{~cm}^{2} / \mathrm{s}$. The migration of the band is towards the positive electrode which is expected for electrophoretic flow of a negatively charged species with suppressed EOF.

The simulations can also be used to extract parameters from the experimental data. Figure 6 shows a graph of the simulated band width at two distinct times $(t=0.99 \mathrm{~s}$, triangles, and $\mathrm{t}=1.98 \mathrm{~s}$, squares) as a function of predicted diffusivities. We were able to extract a diffusivity of the uncaged fluorophore in the microchannel, $\mathrm{D}=1.3 \times 10^{-6} \mathrm{~cm}^{2} / \mathrm{s}$, by comparing the experimental bandwidths (circles) at $t=1.98 \mathrm{~s}$ and $\mathrm{t}=0.99 \mathrm{~s}$ with the calculated curves. This number seems reasonable considering other reported numbers for diffusivity, $\mathrm{D}_{\text {sucrove }}=$
$5.21 \times 10^{-5} \mathrm{~cm}^{2} / \mathrm{s}$ (at $298 \mathrm{~K}$ ) [9] (sucrose has 60\% the mass of fluorescein) and $D_{\text {fluorewein }}$ was reported to be $3.3 \times 10^{-6} \mathrm{~cm}^{2} / \mathrm{s}$ by polarography (experimental conditions unknown) $[4]$. The velocity of the uncaged dye was extracted from the experimental data by plotting the distance versus time for a fluorescent plug moving in an applied field (extracted from experimental images like those shown in Fig. 5). A linear regression was used to obtain the slope or velocity of the line. Velocities at several voltages were obtained in this way and plotted versus the field strength. In the absence of electroosmotic flow, the slope of the line is equal to the electrophoretic mobility $(\mu)$. The mobility was estimated to be $2.3 \times 10^{-4} \mathrm{~cm}^{2} / \mathrm{Vs}$, using the linear region of the graph $(\mathrm{E}>60 \mathrm{~V} / \mathrm{cm})$. The plot shows a non-linear behavior between velocity and field strength for $E<60 \mathrm{~V} / \mathrm{cm}$. We suspect this is not an interaction between the dye molecules and the solvent because the dye is in such low concentration compared to the solvent. A more likely explanation might be the error associated with obtaining measurements at low field strengths when the diffusion velocity is approximately the same order of magnitude as the convective velocity. The extracted diffusivity and mobility from the elastomer straight channels was used successfully in modeling the plug profile in the glass $\mathrm{u}$-shaped channels implying that these parameters can be used to predict flow in a variety of structures.

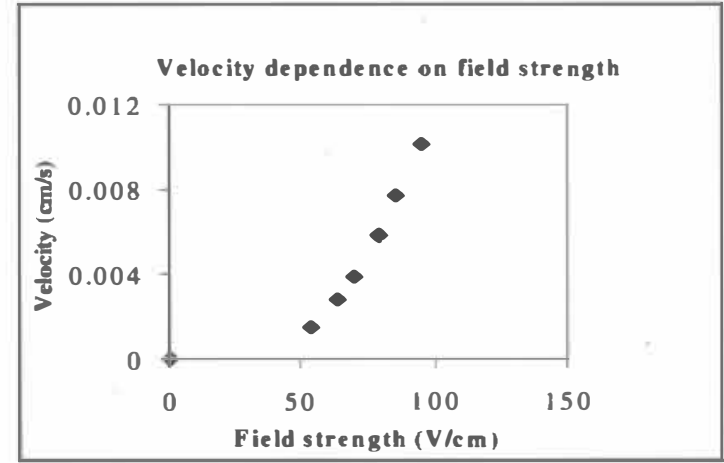

Figure 7. A plot of the velocity vs. field strength used to obtain the mobility of uncaged fluorescein.

Although the field strengths used here are typical for DNA electrophoresis $(\sim 100 \mathrm{~V} / \mathrm{cm})$, the diffusivity of the caged fluorophore in aqueous solution is much higher than that of DNA because of its lower molecular weight (m.w.caged fuorescein $=$ 827 d, m.w. DNA several hundred kd). Therefore, the band profile is dominated by diffusion at these field strengths. In order to predict the loss of resolution from diffusion in DNA separation, a lower diffusivity must be used. We have also examined the flow profile in more complex geometries. Figure 8 compares the experimental to simulated fluorescence profile under electrophoresis in a u-shaped glass microchannel at four different time steps. The images corresponds to $E=69.5 \mathrm{~V} / \mathrm{cm}$. The inset shows the current density in the same section of the microchannel. As the figure shows, the higher current density on the inner wall of the bend causes the inner region of the band to move faster than the outer region, resulting in an asymmetric profile and eventual band broadening. The greater distance traveled by the species along the outer wall further adds broadening and asymmetry to the band. As it travels past the ushaped bend the band takes the shape of a parallelogram. In principle, this shape can be corrected by allowing the band to negotiate an equal and opposite turn downstream of this bend. 


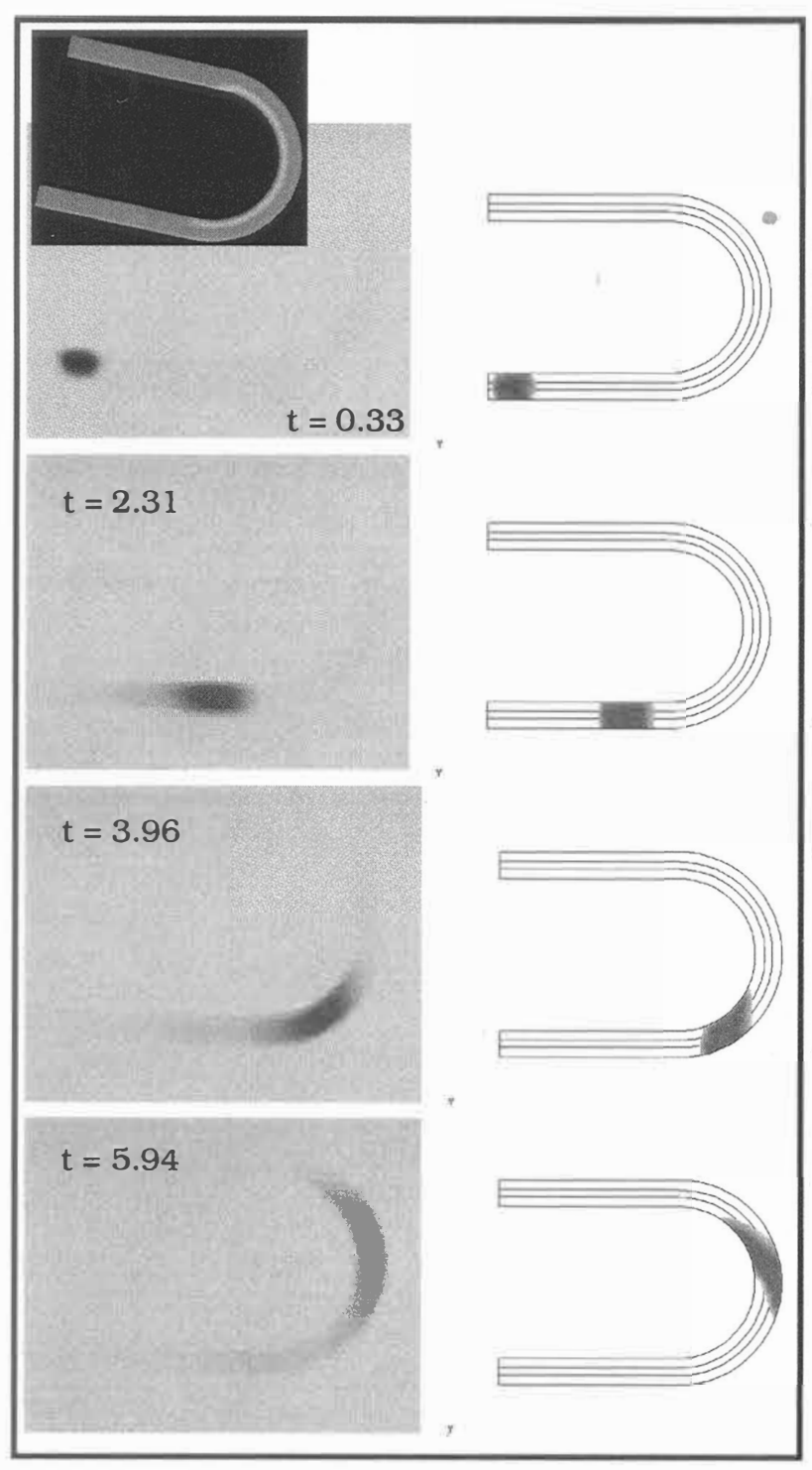

Figure 8. Experimental (left) and simulated (right) electrophoretic flow in a $50 \mathrm{um}$ diameter glass microchannel (radius of curvature $=0.25 \mathrm{~mm}$ ), $E=69.5 \mathrm{~V} / \mathrm{cm}$ (inset: current density in channel, the lightest region corresponds to the highest current density).

However, the broadening effects of the bend and the diffusion cannot be corrected.

The simulation at $\mathrm{E}=69.5 \mathrm{~V} / \mathrm{cm}$ in Fig. 8 closely matched the experiment. As the field strength was increased further the band was observed to take on a parabolic shape that grew increasingly well-defined with increasing field strength. This behavior is contrary to expectations and is not represented in the numerical models. Consequently, the agreement between experiment and simulations falls off at high voltages. Figure 9 shows the experimental band profile at $0.66 \mathrm{~s}$ after uncaging the fluorophore at a range of field strengths, $E=108-125 \mathrm{~V} / \mathrm{cm}$. As the field strength increases above E $100 \mathrm{~V} / \mathrm{cm}$, the parabolic profile becomes more pronounced. The increasing parabolic profile with increasing field strength has also been observed in straight microchannels (data not shown) and straight capillaries [10] and is therefore not due to the asymmetry in the field. The simulations in this field range did not match the experimental data. There are several possible reasons for the observed parabolic shape at high voltages. One possibility is the variation in properties (diffusivity, viscosity, and mobility) across the channel due to joule heating which would cause a temperature gradient in the channel, that becomes more significant at higher voltages. Temperature gradients and their effects on the electrokinetic flow behavior in microchannels have been observed by other researchers [11]. A $1-2^{0} \mathrm{C}$ rise in temperature in the channel can cause the mobility (and viscosity

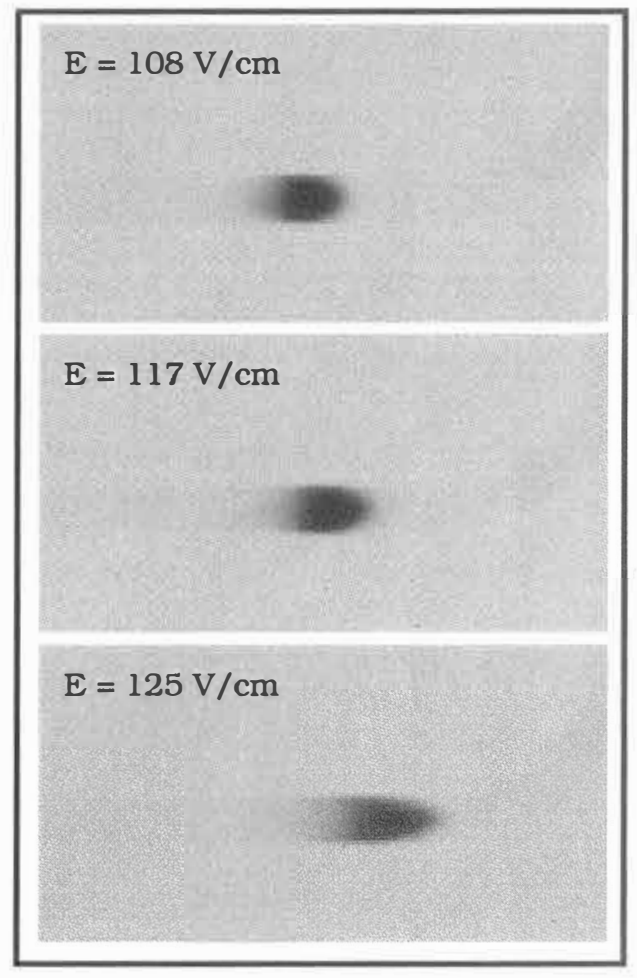

Figure 9. A series of profiles at $t=0.66 \mathrm{~s}$ after the dye was uncaged at varying field strengths.

of the carrier) to change by $2-3 \%$. At higher voltages, where the temperature rise is significant, the change in these material properties may affect the overall flow pattern. A temperature rise may also effect the polymer additive [6], resulting in an incomplete EOF suppression. If EOF is present, then nonuniformities in the zeta potential could induce a pressure gradient and a corresponding parabolic component of the flow. Finally, the parabolic shape may be present at all field strengths but masked by the more rapid decrease in fluorescence at lower field strengths due to diffusion. We are currently exploring these mechanisms to explain the observed deviations from pluglike flow, using both experimental and numerical techniques. An appropriate physical model can then be developed and incorporated into the electrokinetic terms to represent this behavior.

\section{CONCLUSION}

We have used a caged fluorophore to image electrokinetic and pressure-driven flow profiles in both glass and elastomer micrcicharnt?s with varying geometries. Parameters such as diffusivity and mobility were extracted from the experimental data and used in the simulations. This imaging technique has already revealed some interesting and unexpected behavior in these relatively simple fluidic systems. We are looking forward 
to using this method to study these behaviors and extract theoretical models or behavioral models suitable for insertion in modeling tools such as NetFlow.

\section{ACKNOWLEDGEMENTS}

This work was funded, in part, by DARPA (Grant no. F30602-96-2-0306). We would like to acknowledge EG\&G IC Sensors for some of the silicon fabrication, Charles Evans \& Associates for AFM data and the use of the Stanford Nanofabrication Facility.

\section{REFERENCES}

1. P. Paul, D. Rakestraw, and M. Garguilo, "Imaging of pressure- and electrokinetically -driven flow through open capilllaries", Anal. Chem., accepted.

2. J. B. Gravesen, O. S. Jensen, "Microfluidics-a review", $J$. Micromech. Microeng. 3, 168 (1993) and references therein; J. P. Brody and P. Yager, "Low Reynolds number micro-fluidic devices", Solid State Sensors and Actuators Workshop, p. 105, Hilton Head, SC (1996); D. L. Hitt and M. L. Lowe, "Confocal imaging and numerical simulations of converging flows in artificial microvessels", SPIE, 2978, 145 (1997); O. Bakajin, J. Chou, S. S. Chan, J. Knight, L. L. Sohn, R. H. Austin, "Nanoscale structures and flows in biotechnology”, SPIE, San Jose, CA (1998).

3. C. S. Effenhauser, A. Paulus, A. Manz, and H. M. Widmer, "High speed separation of antisense oligonucleotides on a micromachined capillary electrophoresis device", Anal. Chem., 66, 2949 (1994); S. C. Jacobson, R. Hergenroder, L. B. Koutny, and J. M. Ramsey, "High-speed separations on a microchip", Anal. Chem., 66, 1114 (1994); P. Wilding., J. Pfahler, H. Bau., J. N. Zemel, and L. J. Kricka, "Manipulation and Flow of Biological Fluids in Straight Channels Micromachined in Silicon", Clinical Chemistry, 40/1, 43 (1994).

4. A. H. Fan and D. J. Harrison, "Micromachining of capillary electrophoresis injectors and separations on glass chips and evaluation of flow at capillary intersections", Anal. Chem., 66,177 (1994)

5. C. S. Effenhauser, G. J. M. Bruin, A. Paulus, and M. Ehrat, "Integrated capillary electrophoresis on flexible silicone microdevices: Analysis of DNA restriction fragments and detection of single DNA molecules on microchips", Anal. Chem., 69, 3451 (1997).

6. H. M. Wenz, J. Ziegle, D. M. Demorest, J. Stevens, P. M. Williams, and J. W. Efcavitch (unpublished results); H. M. Wenz, "Capillary electrophoresis as a technique to analyze sequence-induced anomalously migrating DNA fragments", Nucleic Acids Res., 22, 4002 (1994).

7. MEMCAD v4.0, Microcosm Technologies, Inc. (www.memcad.com) (1998).

8. G. M. Mala, D. Li, J. D. Dale, "Heat transfer and fluid flow in microchannels", American Society for Mechanical Engineers, 59, 127 (1996); A. Manz, C. S. Effenhauser, N. Burggraf, D. J. Harrison, K. Seller, and K. Flurl, "Electroosmotic pumping and electrophoretic separations for miniaturized chemical analysis systems", J. Micromech. Microeng. 4, 257 (1994); X. C. Qui, L. Hu, J. H. Masliyah, and D. J. Harrison, "Understanding fluid mechanics within electrokinetically pumped microfluidic chips", 1997 International Conference on Solid-State Sensors and Actuators, Chicago, IL (1997).

9. P. W. Atkins, "Physical Chemistry", Third Edition (W. H. Freeman and Company, New York 1986).

10. J. I. Molho, A. E. Herr, T. W. Kenny, M. G. Mungal, P.M. St. John, M. G. Garguilo, D. J. Rakestraw, P. H. Paul, M. Deshpande, and J. R. Gilbert, "Fluid transport mechanisms in microfluidic devices", ASME (1998), in preparation.

11. E. Grushka, R. M. McCormick, and J. J. Kirkland, "Effects of temperature gradients on the efficiency of capillary zone electrophoresis separations", Anal. Chem. 61, 241 (1989) 